\title{
Erratum to: Assessment of postoperative pain after reciprocating or rotary NiTi instrumentation of root canals: a randomized, controlled clinical trial
}

\author{
João Bosko Formigas Relvas ${ }^{1}$ Mariana Mena Barreto Bastos ${ }^{1}$. \\ André Augusto Franco Marques ${ }^{1}$ - Angela Delfina Bitencourt Garrido ${ }^{1}$. \\ Fernando Jose Herkrath ${ }^{2}$ Emílio Carlos Sponchiado Jr ${ }^{1}$
}

Published online: 25 February 2016

(C) Springer-Verlag Berlin Heidelberg 2016

\section{Erratum to: Clinical Oral Investigations}

DOI 10.1007/s00784-015-1692-0

Dr. Fernando Jose Herkrath should be listed as the fifth author. His affiliation is Leônidas \& Maria Deane Institute, Oswaldo Cruz Foundation, Manaus, Brazil. His contributions are as follows: remarkable help in the study design, data analysis, results interpretation and text review. He has no competing interests to declare.

Emílio Carlos Sponchiado, Jr

spemilio@me.com

1 Dental School, Federal University of Amazonas, R Mins Waldemar Pedrosa, n. 1539, Praça 14 de Janeiro, Manaus, AM CEP:

69025-050, Brazil

2 Leônidas \& Maria Deane Institute, Oswaldo Cruz Foundation,

Manaus, Brazil 